\title{
The Impact of Body Language Use of a Conductor on Musical Quality
}

\author{
Bilgen Özcan Çoşkunsoy ${ }^{1}$, Bahar Güdek ${ }^{1}$ \\ ${ }^{1}$ Ondokuz Mayıs University, Faculty of Education Departman of Music Education, Turkey \\ Correspondence: Bahar Güdek, Ondokuz Mayıs University, Faculty of Education Departman of Music Education, \\ Turkey.
}

Received: May 16, 2019

Accepted: June 23, 2019

Online Published: June 30, 2019

doi:10.11114/jets.v7i9.4271

URL: https://doi.org/10.11114/jets.v7i9.4271

\begin{abstract}
In this study, which points out the communication between the choir and conductor, the impact of body language movements of the conductor such as left hand, face, gestures and head on the quality of music in choir is aimed to be analyzed in terms of the fact that as a leader, the conductor, is the most important factor that affects the general music quality of the choir. The study carries an important role as it indicates what affective impacts there can be depending on how the conductor leads "Pulse", "Measure Beat", "Left Hand" and "Body Language" and whether this conductive variety has an impact on musical elements such as Note, Tone Quality / Timbre / Sound, Balance / Sound Stability, Intonation, Rhythm, Articulation / Diction, Vocals, Expression / Emotion, Dynamics, Breath, Synchronization/ Togetherness, Motivation in choir. During the experimental process of the study, a polyphonic piece named "Evening Rise" has been performed by a study group consisted of 29 members who were conducted with the methods of "Pulse", "Measure Beat", "Left Hand" and "Body Language" by the choir conductor. Audial and imagery data records obtained from each conduction method have been converted to Mpeg-4 format and evaluated by 9 expert conductors by using musical sub-dimensions such as Note, Tone Quality, Balance, Intonation, Rhythm, Articulation, Expression, Dynamics, Breath, Synchronization and Motivation that appear in Choir Musical Quality Assessment Form. As an outcome of the research, it is observed that "Left Hand" and "Body Language" conduction methods affect motivation, expression, articulation and dynamics steps placed in Choir Musical Quality Assessment Form a lot and that "Pulse" conduction does not affect musical elements sufficiently and that as a consequence of conduction methods of "Measure Beat", "Left Hand" and "Body Language", choir musical quality has been affected to a large extend.
\end{abstract}

Keywords: body language, choir conducting, musical dynamic, musical quality

\section{Introduction}

In visual communication methods oriented studies which emphasize togetherness of body and movement and musical expression, how musical expression is conceptualized and implemented is of great importance in terms of the assessment of musical performances and development of music education. After defining "what" is expression in music, Abraham (1967) created a platform to discuss "how" it is performed and focused on the expression patterns in music and their use by suggesting that expression is a critical element of a music experience.

According to its oldest definition, choir music which comes from the tradition of singing together and derives from traditional music is an artistic platform that creates an intense communication with choir practices and performances. With the effect of $19^{\text {th }}$ century romantic music movement, conductors who are transformed into music interpreters rather than functioning as a timer made a significant progress about movement and making music and as a result, conductor performances has turned into an interpretation that creates a platform for conductors to express their own intentions to the crowd with their hand, face, position and body movements.

Garretson (1981) declares the fact that conductors are not merely ordinary timers but their fundamental function is to interpret the music for the singers and musicians. These approaches which widen the views of a conductor's role state that conductors should have a broad range of roles including using verbal and non-verbal connection and conductive behaviors. Within the framework of these views, during choir practices and concerts where communication is mostly established in a non-verbal format, there is a constant information flow and conductors convey the composers' musical intention to the choir singers thanks to their own background and body mastery.

As of the very first moment when the conductor comes to the stage, a series of non-verbal interaction starts between the 
conductor, the musicians and the audience. Although this interaction may be perceived as random arm movements and gestures, these body and face movements have a strong impact on musical elements such as choir singers' voices, the rhythm of music, integrity and synchronization of the choir, motivation of singers and the power of expression. This communication is characteristically nonverbal and is demonstrated by both intentional and unintentional behaviors (Fuelbert, 2003, p. 14).

Conductors enable non-verbal communication via hand, arm, face and body movement during a music performance. A conductor communicates through "body language that includes eye contact, body orientation and posture, facial expression, movement of feet, torso, and head, in addition to the expected hand gestures" (Julian, 1989, p. 64). Positioning and balancing the body, circular movements of the body, gestures, eye contact, hand and arm movements are considered to be the whole physical expression used by conductors. While communicating with the choir, primary effect is implemented by hand conduction, and hands which indicate pace and dynamics are also used as a visual stimulant that affect musical expression such as forming sentences, articulation, showing and expressing dynamics, breath and style. However, the use of body and hands which creates the way for a conductor to reach the choir singers through a song differs according to a conductor's conduction ability, communication capacity and the use fundamental non-verbal conduction methods. Rudolf (1950), for instance, emphasizes the importance of the face and eyes in conducting. He states that the expressions conveyed by the conductor's eyes and overall facial expressions relate more to the performers about the conductor's intentions then hand gestures.

Fulberth (2003), who highlights the efficient use of physical activities mentions that bodily movements that a conductor makes with his / her hands, faces, positions and bodies are used to express his / her own musical and voiced intentions to the ensemble. Fulberth also puts forward the fact that if the situation is on the contrary, which means if the conductor uses verbal notice while singing, that affects the performance in a negative way. When considered within the concept of rehearsal, this approach shows that it is more efficient to use non-verbal communication rather than verbalizing specific instructions when the time is valuable. Besides, as non-verbal communication does not distract the choir and interrupt the song, it helps the continuity of the movement, as well.

Mathers (2009) who raised the claim that conductors' body language, general position and facial gestures work better with eye-contact, also argues that gestures can be used as a way to increase the effect of expression and that it is of great importance for a conductor to express his / her musical intention to a group of musicians as impressive as possible. Non-verbal and physical connection that serve as an effective way for conductors to communicate, transform conductors into an interpretive presence and instead of standing as a beat, this connection plays an important role by being the element which reveals the conductor's impressive personality.

This research matters in terms of accepting the fact that a conductor's body movements affect the performance of the choir and music and that these movements are believed to contribute to the improvement of education of the choir. Whether "Pulse", "Measure Beat", "Left Hand" and "Body Language" conducting methods that are named by the conductor have an effect on musical parameters such as Note, Tone Quality / Timbre / Sound, Balance / Sound Stability, Intonation, Rhythm, Articulation / Diction / Vocals, Expression / Emotion, Dynamics, Breath, Synchronization/ Togetherness, Motivation or not is analyzed in this research.

"Pulse" conducting method represents pulse beat, "Measure Beat" represents $4 / 4$ beat pattern with the right hand, "Left Hand" conducting method represents right hand measure beat, left hand, palm goes up, palm goes down, hand parallel to the left and cut move forward, "Body Language" conducting method represents right hand measure beat, left hand palm goes down, palm goes up, left hand parallel and period, cut and body, face, head, gesture and shoulder movements.

In this research, on which level all of these conducting methods affect musical quality and how much these different conducting methods affect parameters that form choir musical quality is aimed to be determined.

\section{Method}

In the research, "Single-group time series pattern", a pattern that the research group is assigned sided, is used so as to identify the effects of the conductor's "Pulse", "Measure Beat", "Left Hand" and "Body Language" conducting ways to the musical quality of the choir in terms of note, tone quality, balance, intonation, rhythm, articulation, expression, dynamics, breath, synchronization and motivation. Time series patterns represent the second biggest category in advanced semi-experiential patterns. In single-group time pattern, it is of primary importance to be sure about keeping the starting level stable. It is preferred to take more than one measurements both during the experience (practice) and after the experience in single-group time series pattern (Gliner, Morgan and Leech, 2015). In this research and on the line of this pattern, dependent variable is considered as musicality and independent variable is considered to be the conductor's body language use that is under the control of the researcher. 
Table 1. General View of the Research Model

\begin{tabular}{lcccc}
\hline Group & NRM & \multicolumn{2}{c}{$\mathrm{O}$} & \\
\hline EG & NRM & O1 & O2 & XO3 \\
O4 & & & &
\end{tabular}

NRM: Non-Random Method, O: Test, EG: Experimental Group, X: Practice, O1: Test 1; O2: Test 2; O3: Test 3; O4: Test 4.

\subsection{Participant}

Study group of this research consists of 29 choir performers in total, 19 of which are students in Faculty of Education, Fine Arts Education Music Education Department of Ondokuz Mayis University and 9 of which are graduated students of the same department and 1 of which is an instructor who has been teaching in the same department.

Performers chosen for the study group are made up of people who have at least 3 years of choir experience in terms of having enough choir information and experience to perform the song according to expert views and having enough musical background and timbre required for the acoustic of the song.

\subsection{Experimental Operation Process}

On the first week, the song named "Evening Rise" is practised with notes by the first conductor, the second conductor conducts the choir by using "Pulse" method and during the practice, both the choir and the second conductor are visually and vocally recorded.

On the second week, the first conductor carries out vocal and breathing exercises, while the second conductor conducts the choir by using "Measure Beat" method. During the practice, both the choir and the second conductor are visually and vocally recorded.

On the third week, the first conductor carries out vocal and breathing exercises to the practice group, while the second conductor conducts the choir by using "Left Hand" method. During the practice, both the choir and the second conductor are visually and vocally recorded.

On the forth week, the first conductor carries out vocal and breathing exercises while the second conductor conducts the choir by using "Body Language" method. During the practice, both the choir and the second conductor are visually and vocally recorded.

Vocal records formed as a result of practices turned into an effective choir conducting and by attaching every movement to each other from the first week are turned into Mpeg-4 format that includes vocal and graffic visuals in order to be assessed separately under the titles of "Pulse", "Measure Beat", "Left Hand" and "Body Language" and they are assessed by experts by using "Choir Musicality Assessment Form".

\subsection{Data Collection Tools}

The song named "Evening Rise" is decided to be used in this research, since it has musical dynamics to reveal musical elements in a choir as a result of conductor's conducting methods such as "Pulse", "Measure Beats", "Left Hand" and "Body Language" and because these criteria can be measured by using this method. "Choir Musical Quality Assessment Form" is collected under 11 sub-headings which are the elements that form choir musical quality such as note (music script), tone quality, balance, intonation, rhythm, articulation, expression, dynamics, breath, synchronization and motivation. It is arranged as a Likert scale assessed between the options $1=$ Very Low and $5=$ Very Good. Considering that gaps of the evaluation tool are even [ 4 gaps / 5 options $=0,80$ value of a gap], firstly lower and upper limits of options are determined. Score intervals and levels below are taken into consideration while calculating and assessing the scores of 9 experts participated in the study on note, tone quality, balance, intonation, rhythm, articulation, expression, dynamics, breath, synchronization and motivation. Average score assessments give information about experts' musical assessments according to the title.

In order to define the credibility of the scores given by assessors for the four conducting methods related to 11 sub-headings, techniques such as correlation, comparison of average scores and agreement percentage are used. Accordingly, it is found that correlation coefficients formed regarding to 11 sub-headings for these four conducting methods are between 0,37 and 0,80 . For "Pulse" conducting method, the correlation coefficient is calculated as 0,69 , for "Measure Beat" conducting method it is 0,72 , for "Left Hand" it is 0,63 and lastly for "Body Language" it is 0,84 . These results show that there is a relationship between assessment scores from strong to very strong (Jawlik, 2016, p.132). So as to perform credibility between assessors, averages of the scores given for the second time are compared in terms of 11 sub-headings for each conducting method. As a result, no significant difference is found between general averages ( $>.05)$. 
In other words, there is no significant difference between the score averages given by the scorers for conducting methods of "Pulse", "Measure Beats", "Left Hand" and "Body Language". Finally, agreement percentages between scores given by assessor are calculated and it is found that scores that they give for four conducting methods and 11 sub-headings have agreement percentages between $\% 71,4$ and \%78,6. As a result, it is seen that credibility is achieved between scores related to measuring device.

Table 2. Score intervals and levels

\begin{tabular}{cc}
\hline Option/Level & Score Interval \\
\hline Very low & $1,00-1,80$ \\
Low & $1,81-2,60$ \\
Average & $2,61-3,40$ \\
Good & $3,41-4,20$ \\
Very good & $4,21-5,00$ \\
\hline
\end{tabular}

\subsection{Analysis of Data}

In the process of data analysis, camera and voice records which belong to conducting methods, following the plan of "Pulse" first week, "Pulse" and "Measure Beat" second week, "Pulse", "Measure Beat" and "Left Hand" third week and "Pulse", "Measure Beat", "Left Hand" and "Body Language" fourth week, are saved step by step and gathered under the titles of "Pulse", "Measure Beat", "Left Hand" and "Body Language". These voice and imagery data recordings of choir conductor and choir performances regarding mentioned conducting methods are assessed by 9 experts based on the criteria of note, tone quality, balance, intonation, rhythm, articulation, expression, dynamics, breath, synchronization and motivation stated in Musical Quality Assessment Form. Each movement and step in the processed data is analyzed as a measurement by using SPSS statistics package software for Windows 23.0. Before starting to analyze the data, whether it is within the boundaries of Musical Quality Assessment Form without any mistakes or deficiencies or not is checked. Kolmogorov- Smirnov (K-S) and Shapiro-Wilk tests are also used to examine the normality distribution of the data.

Based on the conducting methods such as "Pulse", "Measure Beat", "Left Hand" and "Body Language" which are also assessed by experts, arithmetic average (X) and standard deviation (ss) values of scores related to Note, Tone Quality/Timbre / Sound, Balance / Sound Stability, Intonation, Rhythm, Articulation / Diction / Vocals, Expression / Emotion, Dynamics, Breath, Synchronization/ Togetherness, Motivation are calculated.

If there is a statistically significant difference between Note, Tone Quality/Timbre / Sound, Balance / Sound Stability, Intonation, Rhythm, Articulation / Diction / Vocals, Expression / Emotion, Dynamics, Breath, Synchronization/ Togetherness, Motivation, according to what "Pulse", "Measure Beat", "Left Hand" and "Body Language" conducting methods are assessed, is analyzed by non-parametric KruskalWallis test, as normality assumption cannot be reached. When there is a difference noticed between conducting methods, post -hoc Mann-Whitney $\mathrm{U}$ test is applied to identify which conducting methods have statistically significant difference. For all statistical calculations, significance level is considered as .05 . The results of the analysis are presented in series and with the comments in accordance with the determined purposes of the research. After examining the assessments that are related to "Pulse", "Measure Beat", "Left Hand" and "Body Language" separately and generally, it is analyzed if there is a statistically significant difference between performance assessments according to conducting methods such as "Pulse", "Measure Beat", "Left Hand" and "Body Language".

\section{Results}

3.1 Assessment Findings and Comments on the Effect of Conductor's "Pulse" Conducting Method to the Choir Musical Quality

Table 3. Descriptive Statistics Related to Choir Musical Quality Assessment of Pulse

\begin{tabular}{lllll}
\hline Assessed & $\mathrm{N}$ & $\bar{X}$ & ss & Level \\
\hline Note & 8 & 4,25 & 0,89 & Very good \\
Tone quality & 8 & 3,12 & 0,84 & Average \\
Balance & 8 & 3,75 & 0,71 & Good \\
Intonation & 8 & 3,75 & 0,71 & Good \\
Rhythm & 8 & 4,00 & 0,54 & Good \\
Articulation & 8 & 3,50 & 0,54 & Good \\
Expression & 8 & 2,75 & 0,71 & Average \\
Dynamics & 8 & 2,63 & 0,74 & Average \\
Breath & 8 & 3,38 & 0,92 & Average \\
Synchronization & 8 & 3,75 & 0,71 & Good \\
Motivation & 8 & 2,88 & 0,64 & Average \\
\hline
\end{tabular}


According to Table 3, "Musical Quality" assessments of "Pulse" conducting method are generally 'average' and 'good'. Experts assess only one of the musical quality assessment criteria with a high score ( $X$ Note $=4,25 \pm 0,89$; 'Very Good' level), and they assess five criteria as 'average' and five criteria as 'good'.

3.2 Assessment Findings and Comments on the Effect of Conductor's "Measure Beat" Conducting Method to the Choir Musical Quality.

Table 4. Descriptive Statistics Related to Choir Musical Quality Assessment of Measure Beat

\begin{tabular}{lllll}
\hline Assessed & $\mathrm{N}$ & $\bar{X}$ & ss & Level \\
\hline Note & 8 & 4,38 & 0,52 & Very good \\
Tone quality & 8 & 4,25 & 0,46 & Very good \\
Balance & 8 & 4,50 & 0,54 & Very good \\
Intonation & 8 & 4,13 & 0,35 & Good \\
Rhythm & 8 & 4,38 & 0,52 & Very good \\
Articulation & 8 & 4,13 & 0,64 & Good \\
Expression & 8 & 3,87 & 0,35 & Good \\
Dynamics & 8 & 4,13 & 0,35 & Good \\
Breath & 8 & 4,13 & 0,35 & Good \\
Synchronization & 8 & 4,13 & 0,35 & Good \\
Motivation & 8 & 3,87 & 0,35 & Good \\
\hline
\end{tabular}

According to Table 4, "Musical Quality" assessments of "Measure Beat" conducting method are generally 'average' and 'good'. According to Table The highest score belongs to "Note" with a level of 'good' and "Synchronization" and "Rhythm" assessments are also close to "Note". These scores indicate that "Measure Beat" conducting method affects timewise and rhythmic effect in a positive way. The lowest score belongs to "Expression" and "Dynamics" with a level of 'Average'.

3.3 Assessment Findings and Comments on the Effect of Conductor's "Left Hand" Conducting Method to the Choir Musical Quality

Table 5. Descriptive Statistics Related to Choir Musical Quality Assessment of Left Hand

\begin{tabular}{lllll}
\hline Assessed & $\mathrm{N}$ & $\bar{X}$ & ss & Level \\
\hline Note & 8 & 4,38 & 0,52 & Very good \\
Tone quality & 8 & 4,25 & 0,46 & Very good \\
Balance & 8 & 4,50 & 0,54 & Very good \\
Intonation & 8 & 4,13 & 0,35 & Good \\
Rhythm & 8 & 4,38 & 0,52 & Very good \\
Articulation & 8 & 4,13 & 0,64 & Good \\
Expression & 8 & 3,87 & 0,35 & Good \\
Dynamics & 8 & 4,13 & 0,35 & Good \\
Breath & 8 & 4,13 & 0,35 & Good \\
Synchronization & 8 & 4,13 & 0,35 & Good \\
Motivation & 8 & 3,87 & 0,35 & Good \\
\hline
\end{tabular}

According to Table 5, "Music Quality" assessment results of "Left Hand" conducting method are higher and more positive than "Pulse" and "Measure Beat" assessments in general. Experts have assessed four criteria as 'Very good' and the other seven criteria as 'Good'. Results have shown that the increase of conductor's conducting movements affects musical criteria in a positive way. 
3.4 Assessment Findings and Comments on the Effect of Conductor's "Body Language” Conducting Method to the Choir Musical Quality

Table 6. Descriptive Statistics Related to Choir Musical Quality Assessment of Body Language

\begin{tabular}{lllll}
\hline Assessed & $N$ & $\bar{X}$ & $s s$ & Level \\
\hline Note & 8 & 4,75 & 0,46 & Very good \\
Tone quality & 8 & 4,63 & 0,52 & Very good \\
Balance & 8 & 4,63 & 0,52 & Very good \\
Intonation & 8 & 4,63 & 0,52 & Very good \\
Rhythm & 8 & 4,63 & 0,52 & Very good \\
Articulation & 8 & 4,75 & 0,46 & Very good \\
Expression & 8 & 5,00 & 0,00 & Very good \\
Dynamics & 8 & 4,88 & 0,35 & Very good \\
Breath & 8 & 4,63 & 0,52 & Very good \\
Synchronization & 8 & 4,63 & 0,52 & Very good \\
Motivation & 8 & 5,00 & 0,00 & Very good \\
\hline
\end{tabular}

According to Table 6, "Musical Quality" assessment results of "Body Language" are in 'Very good' level in general. Experts have assessed all of the sub-titles as 'Very good' which indicates a high and positive assessment.

3.5 Assessment Findings and Comments Related to Choir's Musical Quality Comparison Depending on The Conductor's Conducting Method

Table 7. Kruskal-Wallis Test Results Regarding Choir Conducting Methods Comparison

\begin{tabular}{|c|c|c|c|c|c|}
\hline Musical Elements & $X^{2}$ & $s d$ & $p$ & Significant Difference & Effect Power \\
\hline Note & 5,85 & 3 & 0,119 & - & - \\
\hline Tone quality & 18,68 & 3 & $0,000 * * *$ & 1,2 and 3,4 & 0,60 \\
\hline Balance & 14,86 & 3 & $0,002 * *$ & 1,2 and 3,4 & 0,48 \\
\hline Intonation & 12,73 & 3 & $0,005 * *$ & $\begin{array}{l}1,2,3 \text { and } 4 \\
2 \text { and } 3\end{array}$ & 0,41 \\
\hline Rhythm & 8,24 & 3 & $0,039^{*}$ & $\begin{array}{l}1,2,3 \text { and } 4 \\
2 \text { and } 3\end{array}$ & 0,27 \\
\hline Articulation & 15,57 & 3 & $0,001 * *$ & $1,2,3$ and 4 & 0,50 \\
\hline Expression & 24,62 & 3 & $0,000 * * *$ & $\begin{array}{l}1,2 \text { and } 3,4 \\
3 \text { and } 4\end{array}$ & 0,79 \\
\hline Dynamics & 24,16 & 3 & $0,000 * * *$ & $\begin{array}{l}1,2 \text { and } 3,4 \\
3 \text { and } 4\end{array}$ & 0,78 \\
\hline Breath & 11,66 & 3 & $0,009 * *$ & $\begin{array}{l}1,2,3 \text { and } 4 \\
1 \text { and } 3\end{array}$ & 0,36 \\
\hline Synchronization & 8,79 & 3 & $0,032^{*}$ & $1,2,3$ and 4 & 0,28 \\
\hline Motivation & 23,76 & 3 & $0,000 * * *$ & $\begin{array}{l}1,2 \text { and } 3,4 \\
3 \text { and } 4\end{array}$ & 0,77 \\
\hline
\end{tabular}

$* p<.05, * * p<.01, * * * p<.001$

$1=$ Pulse, 2= Measure Beat, $3=$ Left Hand and 4= Body Language

Comparisons related to assessments of 11 sub-headings about "Pulse", "Measure Beat", "Left Hand" and "Body Language" conducting methods assessed by experts are done using Kruskal-Wallis H test and then effects of these conducting methods on 11 sub-headings are analyzed with $\eta 2$ (eta) (Table 7). If a significant difference is detected after 
Kruskal-Wallis test, effect size is calculated as $\eta 2$ (eta) and while assessing the effect size, .10 is considered as a 'small' effect, .24 as 'medium', .37 as 'big' and .45 as 'very big' (Leech, Barrett and Morgan, 2005, p.56). Accordingly, different conducting methods do not cause a difference while assessing the choir in terms of notes ( $p>.05)$.

Considering the quality of the tone of the choir, conducting methods cause a significant difference $[X 2(3)=16,68$ and $\mathrm{p}<.001]$. "Left Hand" and "Body Language" conducting methods are scored higher than "Pulse" and "Measure Beat" (assesses as more sufficient). The effect of conducting methods on tone quality is discovered to be very big $(\eta 2=0,60)$.

Conductor's conducting method causes a significant difference on choir balance [X2(3)=14,86 and $\mathrm{p}=0,002]$. "Left Hand" and "Body Language" conducting methods are scored higher than "Pulse" and "Measure Beat" (assessed as more sufficient). The effect of conducting methods on balance is discovered to be very high ( $\eta 2=0,48)$.

Conductor's conducting method causes a significant difference on the choir's intonation $[X 2(3)=12,73$ and $p=0,005]$ and the effect of conducting method on intonation is discovered to be big $(\eta 2=0,41)$. Considering the differences between conducting methods;

a) "Body Language" conducting method is scored higher than "Pulse", "Measure Beat" and "Left Hand" and

b) "Left Hand" conducting method is scored higher than "Measure Beat".

Conducting method causes a significant difference on the rhythm of the choir [X2(3)=8,24 and $\mathrm{p}=0,039]$ and the effect of conducting method on the rhythm is discovered to be on medium-level $(\eta 2=0,27)$. Considering the differences between conducting methods;

a) "Body Language" conducting method is scored higher than "Pulse", "Measure Beat" and "Left Hand" and

b) "Left Hand" conducting method is scored higher than "Measure Beat".

Conducting method causes a significant difference on the articulation of the choir [X2(3) $=15,57$ and $p=0,001]$ and the effects of the conducting method on articulation is discovered to be very big $(\eta 2=0,50)$. Considering the differences between conducting methods; "Body Language" conducting method is scored higher than "Pulse", "Measure Beat" and "Left Hand".

Conducting method causes a significant difference on the expression of the choir $[\mathrm{X} 2(3)=24,62$ and $\mathrm{p}<.001]$ and the effect of the conducting method on expression is discovered to be very big $(\eta 2=0,79)$. Considering the differences between conducting methods;

a) "Left Hand" and "Body Language" conducting methods are scored higher than "Pulse", "Measure Beat" and

b) "Body Language" conducting method is scored higher than "Left Hand".

Conducting method causes a significant difference on the dynamics of the choir [X2(3)=24,16 and $\mathrm{p}<.001]$ and the effect of conducting method on dynamics is discovered to be very big $(\eta 2=0,78)$. Considering the differences between conducting methods;

a) "Left Hand" and "Body Language" conducting methods are scored higher than "Pulse", "Measure Beat" and

b) "Body Language" conducting method is scored higher than "Left Hand".

Conducting method causes a significant difference on the breath of the choir [X2(3)=11,66 and $p=0,009]$ ] and the effect of conducting method on breath is discovered to be on medium-level $(\eta 2=0,36)$. Considering the differences between conducting methods;

a) "Body Language" conducting methods is scored higher than "Pulse", "Measure Beat" and "Left Hand" and

b) "Left Hand" conducting method is scored higher than "Pulse".

Conducting method causes a significant difference on the synchronization of the choir $[\mathrm{X} 2(3)=8,79$ and $\mathrm{p}=0,032]$ and the effect of conducting method on synchronization is discovered to be on medium-level $(\eta 2=0,28)$. Considering the differences between conducting methods; "Body Language" conducting method is scored higher than "Pulse", "Measure Beat" and "Left Hand".

Finally, conducting method causes a significant difference on the motivation of the choir [X2(3)=23,76 and $\mathrm{p}<.001]$ and the effects of conducting method on motivation is discovered to be very big $(\eta 2=0,77)$. Considering the differences between conducting methods;

a) "Left Hand" and "Body Language" conducting methods are scored higher than "Pulse", "Measure Beat" and

b) "Body Language" conducting method is scored higher than "Left Hand". 


\section{Discussion}

Data collected from this research indicates that the effect of "Pulse" conducting method to the choir's musical quality is less than other conducting methods. "Pulse" method, which is the first communication method that the conductor establishes with the choir and which is also the easiest conducting method, is not an expressional conducting method and that is why it cannot create a dramatic change in the musical quality of the choir. In this respect, it would be more suitable to evaluate "Pulse" method as an assistant conducting method that contributes to fundamental choir practices like decoding of a song, sound and lyrics compatibility, sound balance and uniformity studies.

Research findings show that "Measure Beat" conducting method affects choir musical criteria on a medium-level and at a Fgood level and that its effect on note, synchronization and rhythm parameters should be better. For this conducting method which shows the rhythmic pattern of the song, it can be a sufficient conducting method as the conductor can transfer the sound, word and breath balance to the choir by using the right hand to practice the rhythmic pattern of the song in a fundamental frame.

"Measure Beat" is important as it enables choir to balance sound and breath and practice sentence endings, links and sentence integrity responsively with the conductor's beat by reading the notes or performing with words. Measure beat which is also important for basic conductor education is a study dynamics for conductor candidates to perform hand and arm movements. Conductor's performance of this method without any body movements enables choir to perceive the rhythmic harmony of the song and to perform the song without having any musical concern.

Research findings also show that "Left Hand" conducting method's effect on choir musical criteria is at a better level than "Pulse" and "Measure Beat" methods. A conductor who is able to manage using a left hand effectively can ask for musical quality dynamics from the choir with ease. Left hand down and left hand up methods affect not only nuance but also breath, articulation, balance and timbre, content and expression.

According to research findings, "Body Language" conducting method affect choir musical quality criteria on a very good level. "Body Language" which is an expressive method as a whole is accepted to be an efficient conducting method in terms of its great effect o musical criteria required from the choir.

As a result of the findings about the comparison of choir's musical quality depending on the conductor's conducting method, it is found that different conducting methods do not cause a difference while assessing the choir related to notes.

It is observed that "Left Hand" and "Body Language" conducting methods are assessed as better than "Pulse" and "Measure Beat" related to choir's tone quality, balance, intonation, rhythm, articulation, breathing synchronization and motivation.

Imitation of the hand movements, gestures and body movements including the conductor's "Pulse"," Measure Beat", "Left Hand" and "Body Language" methods by the choir performers appears as a significant factor for performance to reach the intended results. Many educators who think that this situation can create practical results for the education of choir music educators and also choir conduction text books focus on the fact that a conductor's musical aim is the clearness and effectiveness of gestural words. This approach can frequently be seen in the studies of Platte (2016), Fulberth (2003) and Grady (2014). These studies focus on the positive effects on musical parameters such as a conductor's body movements on vocal output, choir timbre and increase of the expression power of the choir, sound integrity and intonation.

Musical effects of conducting methods can change depending on the performance and communication skills, music coordination and musical background and most importantly experience of the conductor. It can be seen in the studies of Litman (2006) that non-verbal communication results in less or more musical responds. Besides, Mantemach (2012) states that when choir performers mimic the conductor's movements or behave in a parallel way, a variability shows up in terms of dynamics and musical structure.

Lamb's (2010) studies which emphasizes the importance of gestures while creating movements and music and Durant's (2003) "Choir conduction, Philosophy and Practice" book and his studies about the conductor's body movements and assessment of these movements in terms of choir music can be introduced as examples to the studies that focus on the clearness and efficiency of gestural words and how these musical elements are affected from these gestures and conducting methods.

The results of the research show that musical effect managed by using musical elements which present the choir musical quality such as Note, Tone quality, Balance, Intonation, Rhythm, Articulation, Expression, Dynamics, Breath, Synchronization and Motivation together and in a correct way happens with the conductor's efficient and expressional conducting way.

Choir conduction text books draw attention to conductor's methods, hand and arm movements and leadership features while developing musical intentions of a choir. 
In accordance with this study, "Pulse" and "Measure Beat" methods can have a part in the literature with the explanation that they can be used while decoding a song and managing note and lyrics harmony.

So as to encourage the choir performers, to create productive studies and to present a high quality art, it can be aimed to create a place in the literature by reviewing some gestural words used in the conducting methods "Left Hand" and "Body Language" and some movements together with certain conducting movements.

Categorizing gestures by enriching them for certain musical expectations and performances can not only make musical expression easier but also present a conductive resource to the conductor. This conducting method and expression can be transformed as a developing method.

When gestures play a potential stimulant role in the process of vocalization to perform in a correct and effective way, motivation and expression power of the choir can increase. This situation increases the participation and attention in the choir. In this respect, gestures should take a part in choir conduction related books and programs as visual and textual methods.

\section{References}

Abraham, A. S. (1967). Aesthetics: Dimensions for music education. Music Educators National Conference, Washington, US.

Durrant, C. (2003). Choral conducting: Philosophy and practice. New York: Routledge.

Fuelberth, R. J. V. (2003). The effect of conducting gesture on singers' perceptions of inappropriate vocal tension: A pilot study. International Journal of Research in Choral Singing, 1(1), 13-21.

Garretson, R. L. (1981). Conducting choral music. Boston: MA: Allyn and Bacon, Inc.

Gliner, J. A., Morgan, G. A., \& Leech, N. L. (2015). Research Methods in Practice: Design and Analysis Integrating Approach (Trans. Ed.: Selahattin Turan). Ankara: Nobel Publication Distribution. https://doi.org/10.4324/9781315723082

Grady, M. L. (2014). The effects of nonverbal behaviors exhibited by multiple conductors on the timbre, intonation, and perceptions of three university choirs, and assessed relationships between time spent in selected conductor behaviors and analyses of the choirs' performances (doctoral dissertation). University of Kansas, Kansas. Retrieved from http://hdl.handle.net/1808/15512

Jawlik, A. (2016). Statistics from A to Z. New Jersey, NY: John Wiley \& Sons, Inc.

Julian, J. D. (1989). Nonverbal communication and the conductor. The Instrumentalist, 35, 64-66.

Lamb, G. (2010). Choral techniques. http:// cnx. org/ content/ col11191/1.1

Litman, P. (2006). The relationship between gesture and sound: A pilot study of choral conducting behavior in two related settings. Visions of Research in Music Education, 8, 1-27. http://www-usr.rider.edu/ vrme/v8n1/vision/Litman_Article.pdf

Manternach, J. N. (2012). The effect of varied conductor preparatory gestures on singer upper body movement. Journal of Music Teacher Education, 22 (1), 20-34. https://doi.org/10.1177/1057083711414428

Mathers, A. (2009). The use of gestural modes to enhance expressive conducting at all levels of entering behavior through the use of illustrators, affect displays and regulators. International Journal of Music Education, 27(2), 143-153. https://doi.org/10.1177/0255761409102322

Platte, S. L. (2016). The maestro myth - exploring the impact of conducting gestures on the musician's body and the sounding result. (Post Graduate Thesis). http://opera.media.mit.edu/publications/platte_ms_thesis_2016_maestro_myth.pdf

Rodolf, M. (1950). The grammar of conducting. New York: G. Schirmer.

\section{Copyrights}

Copyright for this article is retained by the author(s), with first publication rights granted to the journal.

This is an open-access article distributed under the terms and conditions of the Creative Commons Attribution license which permits unrestricted use, distribution, and reproduction in any medium, provided the original work is properly cited. 